\title{
The evolution of hydrocarbon dust grains in the interstellar medium and its influence on the infrared spectra of dust
}

\author{
M. S. Murga, ${ }^{1, *} \quad$ S. A. Khoperskov, ${ }^{1,2,3}$ D. S. Wiebe ${ }^{1}$ \\ ${ }^{1}$ Institute of Astronomy, Russian Academy of Sciences, \\ ul. Pyatnitskaya 48, Moscow, 119017 Russia \\ ${ }^{2}$ Universitá degli Studi di Milano, Dipartimento di Fisica, via Celoria 16, I-20133 Milano, Italy \\ ${ }^{3}$ Sternberg Astronomical Institute, Lomonosov Moscow State University, \\ Universitetskii pr. 13, 119992 Moscow, Russia
}

\begin{abstract}
We present evolutionary calculations for the size and aromatization degree distributions of interstellar dust grains, driven by their destruction by radiation, collisions with gas particles, and shattering due to grain-grain collisions. Based on these calculations we model dust emission spectra. The initial grain size distribution play the important role in the evolution of an ensemble of dust particles. Radiation in the considered intensity range mostly aromatizes grains. The smallest grains are mainly destructed via sputtering by collisions with gas particles. There are no grains smaller than $20 \AA$ in the medium at relative gas-dust velocities more than $50 \mathrm{~km} / \mathrm{s}$, which is typical for shocks in supernova remnants. The IR emission spectrum changes significantly due to the dust evolution depending on the adopted grain properties, in particular, on the energy of the $\mathrm{C}-\mathrm{C}$ bonds $\left(E_{0}\right)$. Aromatic bands in the near-IR $(2-15 \mu \mathrm{m})$ are absent, if $E_{0}$ is low, even when the medium properties correspond to the average interstellar medium in our Galaxy. As in reality these bands are observed, high $E_{0}$ values are more preferable. We consider dependence of the emission intensity ratios for various photometric bands on the medium properties. The aromatization degree of small grains shows up most strongly in the $I_{3.4} / I_{11.3}$ intensity ratio while the fraction of aromatic grains in the total dust mass influences the $I_{3.3} / I_{70+160}$ ratio.
\end{abstract}

* E-mail: murga@inasan.ru 


\section{INTRODUCTION}

Modern ground- and space-based infrared (IR) telescopes provided us with the abundant observational material about the composition and evolution of the dust component of the interstellar medium (ISM). One of the key steps in developing our understanding of the dust evolution was the discovery of emission in unidentified IR bands [1], which are now commonly which are now commonly ascribed to the presence of so-called polycyclic aromatic hydrocarbons (PAHs) in the ISM. These macromolecules occupy an intermediate position between molecules and grains $[2,3]$. While PAHs do possess the emission properties necessary to explain the observed bands, it has not yet been well established which form they have in the ISM. In particular, it is not clear whether aromatic compounds exist as an independent component of the ISM or are incorporated into grains, having more diverse chemical structure $[4,5]$. In spite of this uncertainty, the abbreviation PAH is widely used to denote the particles which are the sources of these aromatic bands.

Significant contribution into the study of the nature of aromatic bands can be made by investigating the relationship between their properties and the evolution of dust particles under the influence of ultraviolet (UV) radiation, cosmic rays, and other factors. In particular, different distributions of the emission of aromatic dust grains at $8 \mu \mathrm{m}$ and of other large grains at $24 \mu \mathrm{m}$ in so-called "infrared bubbles" [6, 7] associated with regions of ionized hydrogen could provide evidence for the efficient destruction of PAHs by radiation from massive stars [8].

In the interpretation of observations, value of the mass fraction of PAHs in the total dust mass, $q_{\mathrm{PAH}}$, is often used. This value is a measure of the relative contribution of PAHs to the dust component of the ISM. Observational data in both near and far IR [9] are required to estimate this parameter. Generally, observations with the Spitzer and Herschel space telescopes are used. If no Herschel data are available for a particular object, the ratio of the fluxes in the Spitzer 8 and $24 \mu \mathrm{m}$ photometric bands $\left(F_{8} / F_{24}\right)$ can be used as an indicator of the PAH content $[10,11]$. Information about the nature of PAHs (or, more generally, on the nature of the sources of aromatic bands; e.g., [12]) can be hidden in the dependence of $F_{8} / F_{24}$ on the metallicity of a galaxy or an individual ionized hydrogen complex. It was noted in the works of Wiebe et al. [13] and Khramtsova et al. [14] that both this ratio and its characteristic time variations depend on the metallicity in star-forming regions (SFRs). 
Specifically, $F_{8} / F_{24}$ decreases with time in SFRs with solar or higher metallicities, as is expected if PAHs are destroyed by the UV radiation of young stars more efficiently than are larger dust grains. However, the opposite tendency is observed in SFRs with lower metallicity $-F_{8} / F_{24}$ grows with time, as if the relative amount of PAHs were increasing. There is no correlation between the PAH content and age for SFRs of intermediate metallicity.

These results indicate that the evolution of PAHs in SFRs is a more complicated process and it includes several processes, with UV destruction being only one of them. As one option, a model for cosmic dust was presented by Jones et al. [15] (further J13) that takes into account the possible evolution of the optical properties of grains in a SFR. In this model, the sources of emission in IR emission bands ascribed to PAHs could be hydrocarbon grains with an initially aliphatic structure that becomes aromatized under the action of UV radiation.

In [16] (further Paper I), we presented a model for the evolution of an ensemble of hydrocarbon grains that includes aromatization of hydrocarbon particles, along with the more standard processes of destruction by UV radiation and energetic gas particles and shattering in grain-grain collisions. We continue this work in our current study, and present examples of our modeling of the evolution of an ensemble of dust grains in the ISM and corresponding variations in their emission spectra.

\section{MODEL FOR THE EVOLUTION AND METHODS FOR COMPUTING THE DUST EMISSION SPECTRA}

The model we used in our study is described in detail in Paper 1, and we will only list its main characteristics here. It is assumed in the model that, at an initial time (corresponding, for example, to the formation of dust in evolved stars or the onset of star formation in a molecular cloud), the ensemble of dust particles is characterized by specific distributions of the grain sizes and aromatization degrees, with the latter described by the width of the energy band gap of the grain material $E_{\text {gap }}$. During the evolution of the ensemble, these distributions change due to aromatization and dissociation via the absorption of UV photons and collisions with energetic gas particles (non-thermal and thermal ions and electrons) and shattering during collisions between grains. Our goal here mainly is to investigate the evolution of grains in an ISM with a relatively modest density, where the growth of grains 
as a result of accretion of gas and coagulation is not efficient, so that these processes can be neglected in this work. We plan to include such processes in future studies.

Grain sizes are redistributed because of shattering, in particular, increasing the fraction of small grains. Under certain conditions, shattering could be the main origin for small dust grains in the ISM [17]. In the absence of shattering, small grains are rapidly destroyed by photons, ions, and electrons, so that the ISM would be left without any small grains. In the context of our study, this may be the key process leading to the presence of a noticeable amount of aromatic compounds in the ISM, which are responsible for the observed IR emission bands. Even if fragments arising from the destruction of large grains are mainly hydrogenated and initially have an aliphatic structure, they are rapidly aromatized by the UV radiation.

If conditions are such that the rate of aromatization of small grains is comparable to the rate of their formation, we will observe only aromatic grains in the ISM. If the shattering rate for aliphatic grains exceeds the aromatization rate of the fragments, both aliphatic and aromatic dust grains will be observed. Since astrophysical objects usually do not exist under uniform conditions, the relative abundances of dust grains of various types can change even within a single object. Interpretation of IR spectra requires spatially resolved computations of the evolution of grains, simultaneously modeling all important evolutionary processes under specified and possibly varying external conditions. We present here such computations for fixed conditions in the medium, but investigate the sensitivity of the model to changes in these conditions.

The formation and growth of carbonaceous grains in the atmospheres of evolved stars has been described in detail in [18], however there is no consensus about the initial structure of such grains. Theoretical models predict that the formation of grains in envelopes of carbon stars should mainly lead to the synthesis of aromatic particles (see [19] and references therein). On the other hand, a comparison of aromatic and aliphatic bands in IR spectra of young planetary nebulae shows that grains in most of these objects have predominantly aliphatic structure [20]. Before these grains penetrate into the ISM, the balance can shift to a greater predominance of aromatic grains due to the action of the UV radiation of the nucleus of the planetary nebula. We assume in our study that all grains are initially aliphatic, although our model can also handle different initial conditions.

The most important parameters of our model are the gas temperature $T$, the number 
densities of $\mathrm{H}^{+}, \mathrm{He}^{+}$, and electrons, the radiation field, and the velocity of the relative nonthermal motion of the gas and dust, for example, due to the propagation of shocks. We characterize the radiation field using the parameter $U$, equal to the radiation intensity from $912 \AA$ to $2500 \AA$, expressed in units of the corresponding radiation intensity in the solar neighborhood determined in [21]. In other applications our model can use different types of spectra, including those with an appreciable contribution at wavelengths shorter than $912 \AA$.

The dust is described by a two-dimensional distribution over the grain radius $a$ and the parameter $E_{\text {gap }}$, with the number of bins being $N^{\mathrm{a}}$ and $N^{\mathrm{eg}}$, respectively. Although the smallest grains considered in the work have properties more similar to molecules than to dust particles, for the sake of uniformity, we refer to dust particles of any size as grains. Of the parameters describing the evolution of dust presented in Paper 1, we also varied the valus of $E_{0}$ characterizing the energy of the $\mathrm{C}-\mathrm{C}$ bonds. This energy is poorly known, and the computational results depend quite substantially on our choice of its value, making it necessary to treat $E_{0}$ as a parameter of our model.

The method used to compute the evolution of the grain distributions over their size and $E_{\text {gap }}$ is described in detail in Paper I. Here, we also present modeling of spectra for a given ensemble of grains. The emission coefficient $j_{\nu}$ for dust consisting of $N_{\text {type }}$ types of grain heated by a radiation field $U$ was computed using the formula

$$
\begin{aligned}
j_{\nu}= & \sum_{i=1}^{N_{\text {type }}} \int\left(\frac{d n}{d a}\right)_{i} C_{\text {abs }}(i, a, \nu) d a \times \\
& \times \int B_{\nu}(T)\left(\frac{d P}{d T}\right)_{i, a, U} d T
\end{aligned}
$$

where $(d P / d T)_{i, a, U}$ is the temperature distribution for grains of type $i$ with radius $a$ in the radiation field $U$, and $(d n / d a)_{i}$ is the size distribution of grains of type $i$. By various grain types, we mean hydrocarbon grains with various values of $E_{\text {gap }}$ and grains with other chemical compositions, such as silicate grains, which are included in our model for the computation of the spectra.

We take into account the fact that small grains (with radii less than $\sim 250 \AA$ ) are sensitive to single photon absorption, which heat these grains to high temperatures on short time scales, after which they cool rapidly via IR radiation $[22,23]$. An ensemble of such grains in the presence of stochastic heating cannot be characterized by a single temperature, and we need to consider the distribution $d P / d T$, which can be computed using the method 

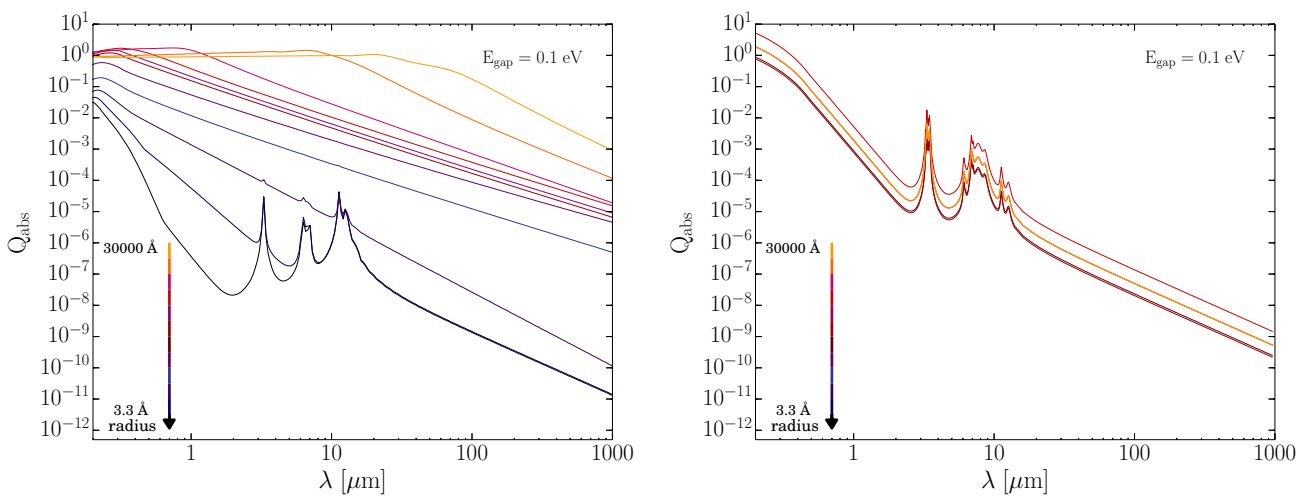

Figure 1. Wavelength dependence of the absorption efficiency $Q_{\text {abs }}$ with fixed $E_{\text {gap }}=0.1 \mathrm{eV}$ for hydrocarbon grains with radii from 3.3 to $30000 \AA$ (the color varies from orange to black with decreasing grain size). The left panel shows results for grains that are fully aromatized and have radii not exceeding $200 \AA$. It is assumed that only an outer layer $200 \AA$ thick of large grains is aromatized.

The right panel shows results for grains that are fully aromatized independent of the size.

presented in $[24,25]$. The temperature of large grains can be found from the condition of thermal balance; the temperature distribution of such grains is a delta function. The heat capacity evaluation for hydrocarbon grains $C_{V}$ is required to calculate their temperature distribution. It was performed as described in Paper 1. The heat capacity of silicate grains was calculated as in [24].

The optical constants (the real and imaginary parts of the refraction index $n$ and $k$ ) of hydrocarbon grains with radii up to $200 \AA$ were taken from [26-28] (see also [29, 30]). We assumed that small grains are aromatized throughout their volume, i.e., they have a uniform aromatization degree. We assume large grains $(a>200 \AA)$ to have two layers: the inner layer consists of hydrogenated, amorphous hydrocarbon material with $E_{\text {gap }}=2.67 \mathrm{eV}$, while the outer layer may be restructured by incident UV radiation, so that, in general, the optical constants for the surface layer may differ from those for the inner layer. We computed the absorption efficiency $Q_{\mathrm{abs}}=C_{\mathrm{abs}} / \pi a^{2}$ for two-layer grains using code based on the Mie theory, described in [31]. The optical properties of silicate grains are taken from [32, 33]. These grains contribute only to the continuum emission at the considered conditions.

The left panel of Fig. 1 shows $Q_{\text {abs }}$ for hydrocarbon grains of various sizes with fixed $E_{\text {gap }}=$ $0.1 \mathrm{eV}$. The behavior of $Q_{\mathrm{abs}}$, and therefore $j_{\nu}$, at wavelengths from 2-20 $\mu \mathrm{m}$ depend strongly on the grain size. Bands are weak for grains with radii of about $200 \AA$, but become stronger 


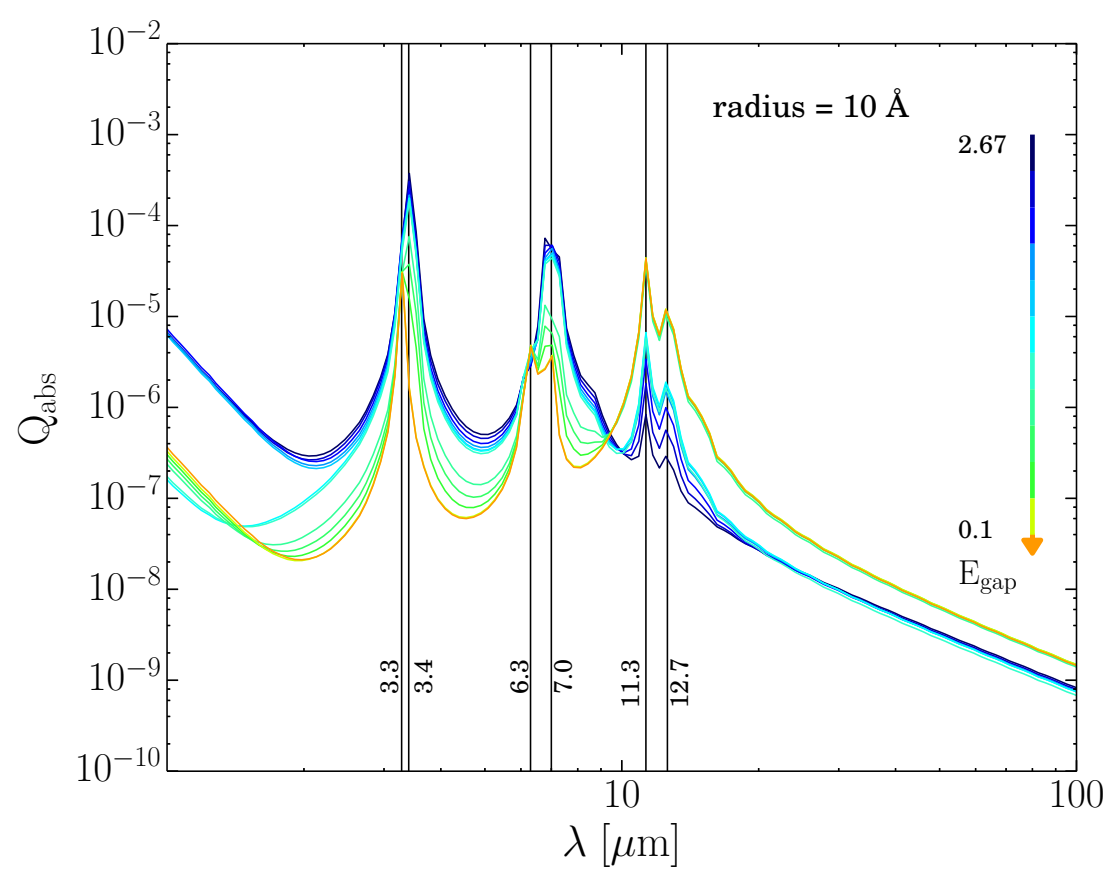

Figure 2. Wavelength dependence of the absorption efficiency $Q_{\text {abs }}$ for grains with radii of $10 \AA$ and $E_{\text {gap }}$ values from 2.67 to $0.1 \mathrm{eV}$ (the color varies from orange to blue with increasing $E_{\text {gap }}$ ).

with decreasing $a$. Since most larger grains are comprised of hydrogenated material, the characteristics of the relatively thin aromatized surface layer are "muffled". For comparison, the right panel of Fig. 1 shows $Q_{\text {abs }}$ for grains of various sizes without any restriction on the aromatization depth (i.e., the grains are fully aromatized regardless of their size).

Figure 2 shows the absorption efficiency of grains with radii of $10 \AA$ and various values of $E_{\text {gap }}$. Predominantly aliphatic ( $E_{\text {gap }}=2.67 \mathrm{eV}$, blue curves) and predominantly aromatic ( $E_{\text {gap }}=0.1 \mathrm{eV}$; orange curves) grains are characterized by peaks in $Q_{\text {abs }}$ at various wavelengths or bands with appreciably different maximum values and widths. The most substantial $Q_{\text {abs }}$ peaks for aromatic compounds are at 3.3,6.3,6.7, 11.3, and $12.7 \mu \mathrm{m}$, while aliphatic compounds have their main peaks at 3.4 and $7.0 \mu \mathrm{m}$, with their $Q_{\text {abs }}$ peaks at 11-12 $\mu \mathrm{m}$ being appreciably weaker.

The ratio of the intensities of the spectral features at 3.3 and $3.4 \mu \mathrm{m}$ is often used to estimate the relative abundance of aromatic and aliphatic compounds [34-36], but obviously spectra with higher resolution are required to separate these features. Moreover, it can be seen in Fig. 2, the bands at 3.3 and $3.4 \mu \mathrm{m}$ may overlap, depending on which grain type dominates. 


\section{EVOLUTION OF THE DISTRIBUTIONS OF GRAIN SIZE AND AROMATIZATON DEGREE}

In this section, we consider variations of the distributions of the size and aromatization degree of hydrocarbon grains. The number density of hydrogen ions $n_{\mathrm{H}^{+}}=1 \mathrm{~cm}^{-3}$, number density of helium ions $n_{\mathrm{He}^{+}}=0.1 \mathrm{~cm}^{-3}$, and temperature $T=10^{4} \mathrm{~K}$ of the medium were fixed in the calculations. The parameter $U$ characterizing the radiation field, which is the main factor influencing the aromatization process, and the gas velocity $v_{\text {ion, }}$, which is a determining factor in the destruction of small dust grains, were varied. As in [37], the relative velocity of the grains used when modeling shattering was taken from [38] for a thermally ionized medium, where the dust dynamics were computed for a turbulent medium using a magnetohydrodynamical model. We used the J13 distribution proposed in [15] as the initial size distribution of the a-C:H grains, unless stated otherwise. This distribution assumes that the partially aromatized hydrocarbon dust consists of small grains with radii from 4 to $1000 \AA$, whose size distribution is a power law with an exponential tail, and large grains with a lognormal size distribution and a characteristic radius of $\sim 2000 \AA$. The J13 distribution also includes silicate grains with a lognormal size distribution and a characteristic radius of $\sim 2000 \AA$. We take into account the presence of silicate grains only when modeling spectra, and assume that the silicate grains are not subject to evolutionary variations. This assumption is justified because the destruction of silicate grains is inefficient under the conditions considered [37].

Figure 3 shows examples of finite grain-size distributions summed up by the aromatization degree for several different cases. In all panels, a dotted black curve shows the initial distribution. To estimate the minimum number of bins required to obtain realistic results, we considered runs with $N^{\mathrm{a}}=15,30$, and 60 and with $E_{0}=5.0 \mathrm{eV}$ after one million years of evolution, shown by blue, green, and red curves in Fig. 3a. The computations for $N^{\mathrm{a}}=30$ and 60 are in good agreement, but reducing $N^{\mathrm{a}}$ to 15 leads to overestimation of the shattering rates for some size bins. This is consistent with the conclusions of Hirashita and Yan [37], who found the optimal number of bins for computations with shattering to be 32. However, in addition to size binning, we also consider several (five in this study) bins in the aromatization degree, so, for example, for $N^{\mathrm{a}}=30$ the total number of bins is 150 , leading to substantial losses in the computation speed for a given accuracy. Therefore, we 

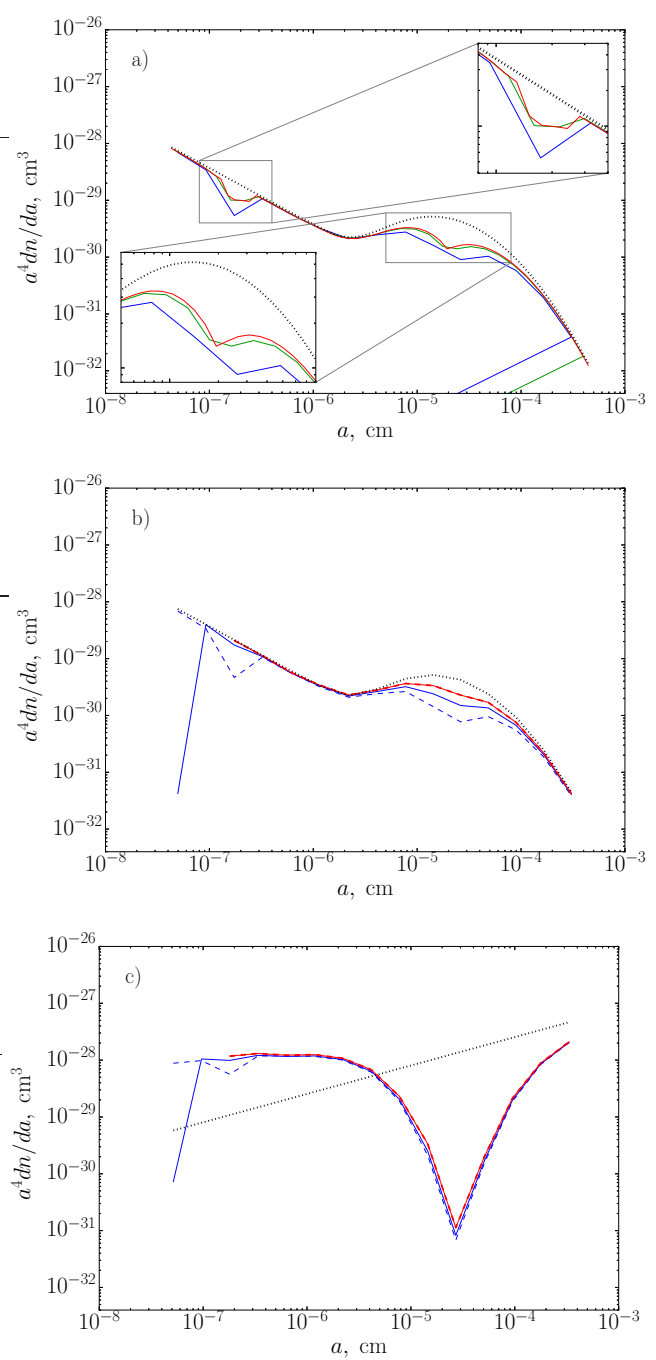

Figure 3. Size distributions of dust grains for various conditions for computations covering one million years of evolution. (a) Red, green, and blue curves show the results of computations for $U=1$ and $v_{\text {ion }}=10 \mathrm{~km} / \mathrm{s}$ with $N^{\mathrm{a}}=60,30$, and 15 , respectively. The initial distribution (J13) is shown by the dotted black curve. (b) Blue curves show the grain size distribution after one million years of evolution for $U=1$ and $v_{\text {ion }}=10 \mathrm{~km} / \mathrm{s}$ for $E_{0}=2.9 \mathrm{eV}$ (solid) and $E_{0}=5.0 \mathrm{eV}$ (dashed). Red curves show the grain size distribution for $U=10^{5}$ and $v_{\text {ion }}=50 \mathrm{~km} / \mathrm{s}$ for $E_{0}=2.9 \mathrm{eV}$ (solid) and $E_{0}=5.0 \mathrm{eV}$ (dashed). The initial distribution (J13) is shown by the dotted black curve. (c)

Same as (b) but adopting the MRN distribution [39] as the initial distribution.

conclude that, when incorporating the model in the computations for the real object in $1 \mathrm{D}$ or even more so in 3D, it is still acceptable to use $N^{\mathrm{a}} \sim 15$, since the errors that arise due to the insufficient number of bins are smaller than evolutionary changes in the distribution. 


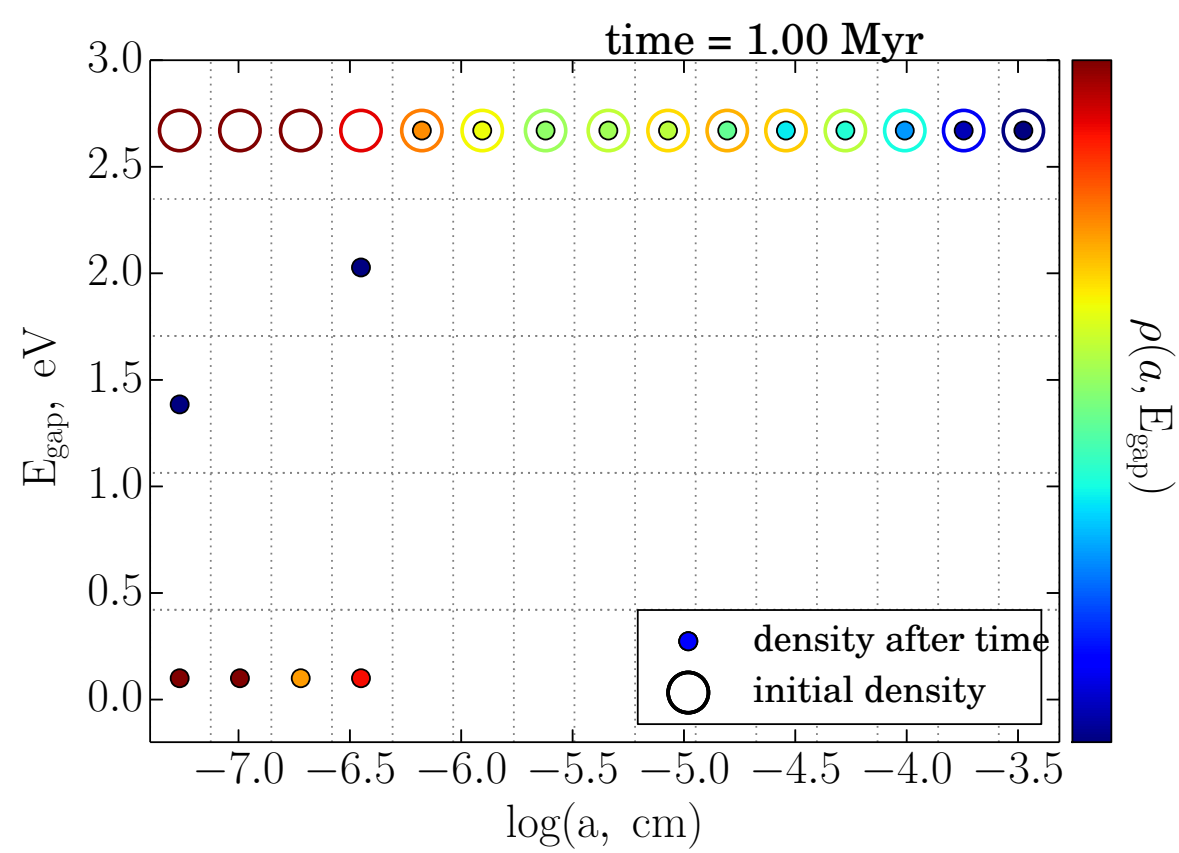

Figure 4. A $15 \times 5$ field separated into cells in the grain radius $a$ and aromatization degree $E_{\text {gap }}$.

If grains are present in the distribution at a given time, a circle is placed in the cell for the corresponding parameters. Otherwise, a cell is empty. The color of the circle corresponds to the mass density $\tilde{\rho}\left(m, E_{\text {gap }}\right)$ for the grains of the given type. Grains of all sizes are unaromatized at the initial time; their positions in the grid are shown by empty circles.

Further, we will present results for our computations with $N^{\mathrm{a}}=15$.

Further, we carried out computations of the grain destruction for parameters typical of the "ordinary" ISM $\left(U=1, v_{\text {ion }}=10 \mathrm{~km} / \mathrm{s}\right)$ and for "extreme" parameters $\left(U=10^{5}\right.$, $v_{\text {ion }}=50 \mathrm{~km} / \mathrm{s}$ ). The results are shown in Fig. $3 \mathrm{~b}$. The blue curves show the grain size distribution after one million years of evolution in an "ordinary" ISM for $E_{0}=2.9 \mathrm{eV}$ (solid) and $E_{0}=5.0 \mathrm{eV}$ (dashed). At the smaller value of $E_{0}$, the efficient destruction leads to a virtual absence of grains with sizes less than $10^{-7} \mathrm{~cm}$. A second important result is the smoothing of the maximum corresponding to large grains $\left(a \sim 10^{-5} \mathrm{~cm}\right)$. The destruction of small grains is not as efficient when $E_{0}=5.0 \mathrm{eV}$, leading to a more substantial reduction in the number of large grains, since the main (essentially the only) mechanism for the destruction of grains in an ambient medium with the specified parameters is collisions with other grains, with small grains playing an important role. Moreover, the efficient destruction of large grains helps to retain the high (compared to the case of $E_{0}=2.9 \mathrm{eV}$ ) 
abundance of the smallest grains.

The results of the evolution of the grain size distribution under the "extreme" conditions are shown by the red curves in Fig. 3b, which also correspond to a computed time of $10^{6}$ yrs. Essentially the only result of this evolution for both considered values of $E_{0}$ with the J13 initial distribution is the efficient destruction of small grains and, accordingly, a reduced destruction of large grains due to the dearth of small grains (the solid curve for $E_{0}=2.9 \mathrm{eV}$ and the dashed curve for $E_{0}=5.0 \mathrm{eV}$ essentially coincide).

Figure 3c shows the grain size distribution computed with an MRN distribution [39] as the initial distribution. The MRN distribution differs from the J13 distribution in having a smaller initial fraction of small grains. In this case, the main factors, changing the distribution, are the shattering of large grains, which leads to a significant increase in the number of small grains, and the destruction of small grains by radiation and/or collisions with gas particles. In the distribution (Fig. 3c), this is expressed through a sharp minimum near radii of $\sim 3 \times 10^{-5} \mathrm{~cm}$, an increase in the number of grains with radii of $\lesssim 3 \times 10^{-6} \mathrm{~cm}$, and the disappearance of grains with radii of $\lesssim 10^{-7} \mathrm{~cm}$. Since the main evolutionary factor for the grain size distribution in the considered case is shattering, whose parameters we have not varied, the results for various values of $E_{0}, U$, and $v_{\text {ion }}$ are all roughly the same.

Another way of presenting results of computations is used in Figure 4. Here we show a grid with cells corresponding to various sizes and aromatization degrees. A cell is occupied at a given time if teher are grains in the model with the corresponding $a$ and $E_{\text {gap }}$ values. Initially, grains of all sizes are fully hydrogenated; i.e., $E_{\text {gap }}=2.67 \mathrm{eV}$. The initial distribution of grains in Fig. 4 is shown by large empty circles in the upper row of the grid. Filled smaller circles correspond to the grain distribution after one million years of evolution with $U=1$ and $v_{\text {ion }}=10 \mathrm{~km} / \mathrm{s}$. The pattern has changed: in the size range $\log a(\mathrm{~cm})<-6.3$, the dust has moved to cells with a higher aromatization degree. The color of the circles in Fig. 4 corresponds to the mass density in a given cell. With the specified parameters and the J13 initial size distribution, most of the bins lose mass, but under other conditions (for example, with the MRN initial distribution [39]), the amount of dust can grow in low mass bins. When other values of $U$ are used, small hydrogenated grains may be preserved in the grid, or, on the contrary, large aromatized grains may appear.

Generally, grain size distribution and $E_{\text {gap }}$ depend on the conditions in the medium, that it, on the radiation field, temperature, and velocities of gas and dust. The external factors 
influence mostly small grains, leading to these grains being "swept out" of the distribution. With typical parameters of the ISM, collisions with gas particles are a more efficient destructive factor, while the radiation field only aromatizes small grains. It is important to remember that, in the considered model, aromatization is a necessary step preceding the efficient grain destruction.

The action of external factors on large grains only shows up in the case of extreme values of the corresponding parameters. "Softer" values of $U$ and $v_{\text {ion }}$ probably facilitate the preservation of large grains, since small grains are efficiently destroyed in this case, so that the shattering of large grains due to collisions with small grains becomes less efficient. We do not present here results of computations for other densities and temperatures, but the conclusions of Paper 1 suggest that these parameters also play an important role. Overall, the action of external factors and interactions between grains could substantially change the parameters of the dust population. The result of this evolution depends appreciably on a combination of many circumstances, which can explain the observed variations in the properties of dust grain ensembles in various regions of the Milky Way and other galaxies. This is supported, in particular, by observations of the extinction curves and other properties of dust in various directions $[40,41]$. Variations in the dust parameters can probably be explained by the variety of external conditions in which the dust resides, which makes it impossible to build a universal model, suitable for any object.

\section{EVOLUTIONARY VARIATIONS OF THE SPECTRAL AND PHOTOMETRIC CHARACTERISTICS OF A DUSTY MEDIUM}

We discussed above how the grain size and aromatization degree distributions can change due to evolutionary processes. However, observations do not allow directly judging the composition and distributions of dust over various parameters. Thus, it is necessary to consider how evolutionary processes influence observed dust spectra. As was noted above, we used the J13 distribution as the initial size distribution for the grains (Fig. 3a, black dotted curve), which satisfies the main observational constraints (IR emission, absorption properties, particularly in the UV, etc.).

Figure 5 shows dust emission spectra at the initial computation time and after one million years for several sets of external conditions. Three parameters were varied: the intensity of 

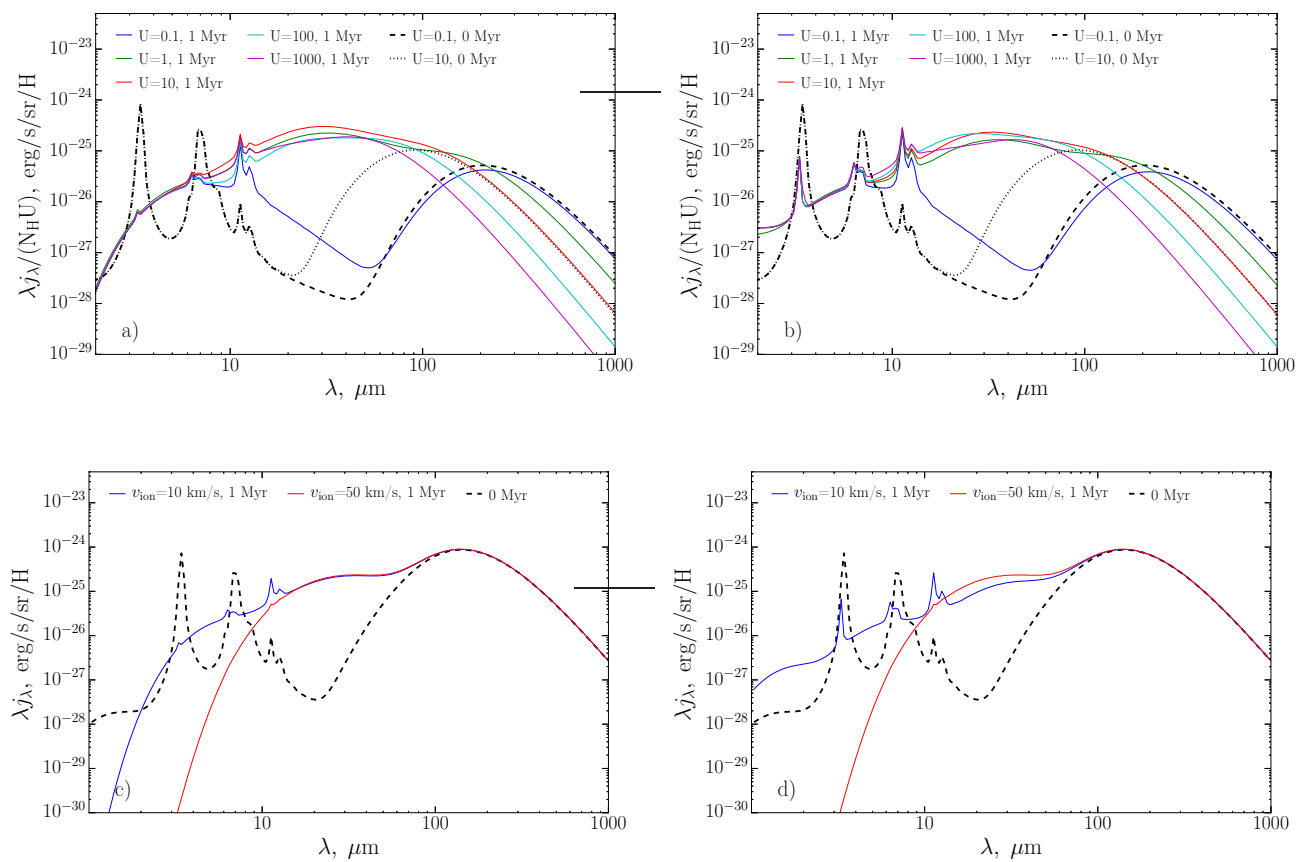

Figure 5. Top left: dust emission spectrum at the initial time in the presence of radiation with an intensity of $U=0.1$ (dashed curve) and $U=10$ (dotted curve), and after one million years for $U=0.1,1,10,100$, and $1000, v_{\text {ion }}=10 \mathrm{~km} / \mathrm{s}$ and $E_{0}=2.9 \mathrm{eV}$. Top right: same for $E_{0}=5 \mathrm{eV}$.

Bottom left: dust emission spectrum at the initial time (dashed curve) and after one million years for velocities of $v_{\text {ion }}=10$ and $50 \mathrm{~km} / \mathrm{s}$, with $U=1$ and $E_{0}=2.9 \mathrm{eV}$. Lower right: same for $E_{0}=5 \mathrm{eV}$.

the radiation field $U$, the velocity of collisions between the gas and dust $v_{\text {ion }}$, and $E_{0}$. The upper panels show how the dust spectrum changes under the action of external radiation fields of various intensities, for a fixed collision velocity of $10 \mathrm{~km} / \mathrm{s}$. The parameter $E_{0}=$ $2.9 \mathrm{eV}$ for the plots in Fig. 5a and $E_{0}=5 \mathrm{eV}$ in Fig. 5b. On all the panels, spectra for the initial size distribution are shown in black, with $U=0.1$ (dashed) and $U=10$ (dotted). They characterize the emission of aliphatic hydrocarbons with strong bands at 3.4 and $7 \mu \mathrm{m}$. The J13 initial distribution has a large quantity of small grains, which are responsible for radiation at $2-20 \mu \mathrm{m}$, making these bands very strong.

After one million years with $E_{0}=2.9 \mathrm{eV}$ (Fig. 5a), even a weak UV radiation field destroys most small grains, and only the features at 11.3 and $12.7 \mu \mathrm{m}$, which are generated by aliphatic grains, remain in the near-IR spectrum. Emission bands corresponding to aromatic compounds are preserved only in the case of higher values of $E_{0}$ parameter, $5 \mathrm{eV}$ (Fig. 5b). Thus, since aromatic bands at wavelengths shorter than $10 \mu \mathrm{m}$ are in fact observed in the 


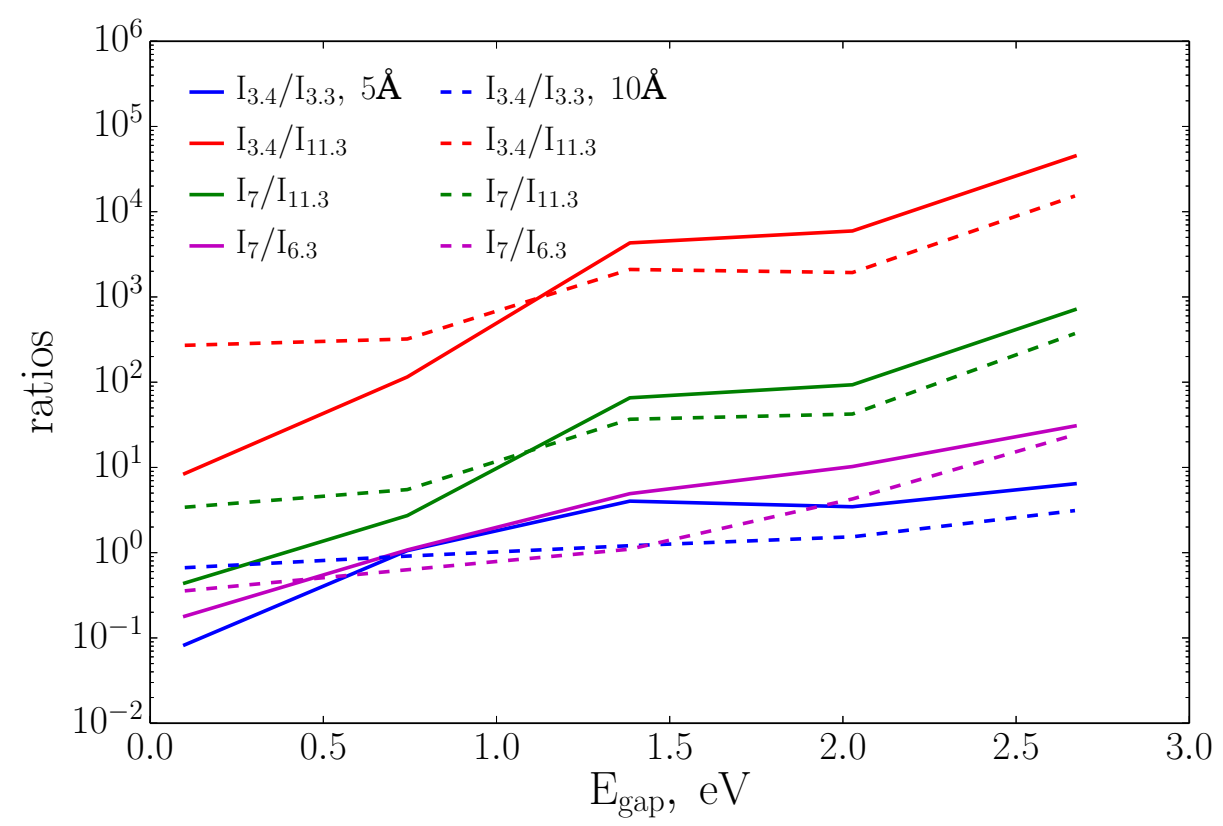

Figure 6. Radiation intensity ratios in some aromatic and aliphatic bands for grains with radii of $5 \AA$ (solid curves) and $10 \AA$ (dashed curves) for $U=1$.

ISM, a higher value of $E_{0}$ seems to be preferred. Variations in the intensity of the radiation field $U$ influence the form of the continuum (in particular, the position of the long-wavelength maximum) only at wavelengths longer than $10 \mu \mathrm{m}$ (in the considered range).

Aromatization is efficient even when $U=0.1$. The computations show that the aromatization rate is appreciably reduced only when $U=0.01$. Such low radiation intensities can be encountered at the peripheries of galaxies, far from stars and SFRs; however, all dust grains with radii smaller than $\sim 100 \AA$ in the vicinities of stars, especially massive stars, will be aromatized on short times scales. Note that the radiation field plays a double role in the formation of spectra, simultaneously disrupting small grains and causing them to radiate, so that the response of the spectra to an increase in the radiation field is not linear.

The lower panels in Fig. 5 show the computed dust spectra for $U=1$ after a million years of evolution and various velocities $v_{\text {ion }}$. Also, as in the upper panels, the black dashed curve shows the spectrum for the initial size distribution. There are no aromatic bands in the spectrum when $E_{0}=2.9 \mathrm{eV}$ and $v_{\text {ion }}=10 \mathrm{~km} / \mathrm{s}$, but there is an appreciable continuum at wavelengths of several microns, indicating the presence of stochastically heated small grains. Appreciable aromatic bands also appear in the spectrum when $E_{0}=5 \mathrm{eV}$. Increasing the 
velocity to $50 \mathrm{~km} / \mathrm{s}$ has substantial consequences: bands in the near IR are not observed for either $E_{0}$ value. This means that, in the presence of such velocities, the rate of destruction of small grains exceeds the rate of their formation.

Thus, we can draw the following conclusions. (1) The intensity of the radiation field is important for aromatization of grains, but, even when $U=1000$, in the absence of IR bands, a substantial continuum is observed at wavelengths of several microns. This means that the smallest aromatic grains are destroyed in this medium, but stochastically heated grains smaller than $250 \AA$ in size are preserved. The efficient destruction of any small grains is the result of either collisions with gas particles with velocities above several tens of $\mathrm{km} / \mathrm{s}$ or the presence of a very strong radiation field. (2) A C-C binding energy of about $3 \mathrm{eV}$ would lead to the rapid destruction of small grains and the disappearance of aromatic bands. Since such bands are observed, the energy $E_{0}$ must be of the order of $5 \mathrm{eV}$ or higher.

Since substantially less spectral data than photometric data on dust emission in the ISM are available, it is interesting to trace how various external conditions are reflected not only in the appearance of the spectrum, but also in ratios of integrated intensities in the near, middle, and far IR. The ratio of fluxes at 8 and $24 \mu \mathrm{m}$ wavelengths, which correspond to the central wavelengths of the Spitzer IRAC and MIPS filters, have often been used to characterize the abundances of small grains and PAHs [42]. The Herschel Space Telescope (PACS instrument) can be used to study dust emission in bands at 70, 100, and $160 \mu \mathrm{m}$ with comparable angular resolution. We computed synthetic intensities in these filters taking into account response functions of the IRAC, MIPS, and PACS instruments ${ }^{1}$. In addition to the intensities at 8, 24,70, and $160 \mu \mathrm{m}$, we also calculated how they are related to the intensities at wavelengths of $3.3,3.4,6.3,7$, and $11.3 \mu \mathrm{m}$. The response functions for these bands were chosen so that they were equal to unity if a feature was located inside the interval and 0 if it was located outside the interval. The $I_{6.3} / I_{70+160}$ and $I_{3.3} / I_{70+160}$ ratios can be used to obtain information about variations in the abundance of small aromatic dust relative to the abundance of large dust grains, while the $I_{3.4} / I_{70+160}$ and $I_{7} / I_{70+160}$ ratios can be used to estimate the abundance of aliphatic dust. Furthermore, we separately considered $q_{\mathrm{PAH}}$, the mass fraction of small aromatic dust grains with radii $a<20 \AA$ and $E_{\text {gap }}<1 \mathrm{eV}$ in the total mass of dust $[10,43]$. We again emphasize that, although the conventional notation used for

\footnotetext{
1 The response functions were taken from http://ssc.spitzer.caltech.edu and http://herschel.esac.eas.int.
} 

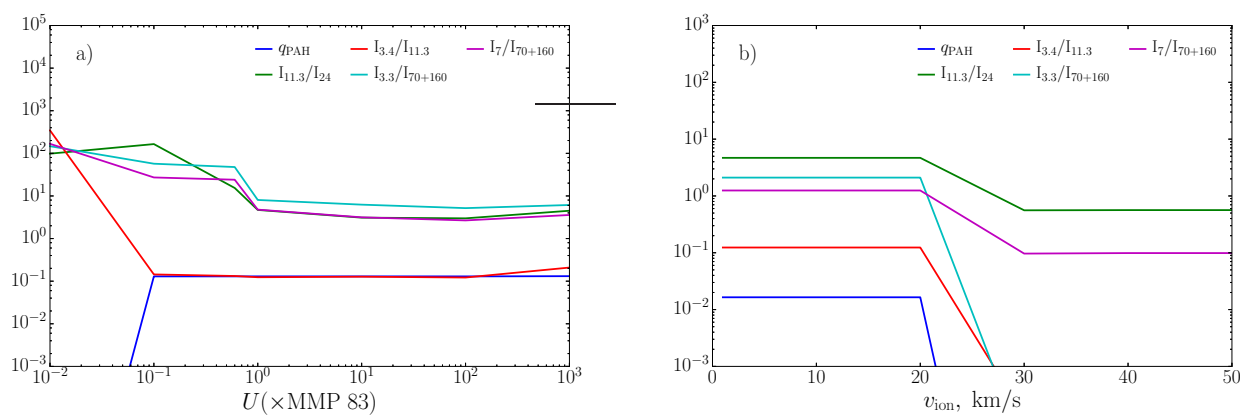

Figure 7. Left: intensity ratios for various wavelengths and mass fractions of small aromatic grains as a function of the radiation field intensity $U$ after one million years of evolution. Right: same, as a function of the velocity of collisions between gas and dust particles for $E_{0}=5.0 \mathrm{eV}$.

these grains is "PAH" (polycyclic aromatic hydrocarbons), we interpret this quantity more broadly, as the fraction of any even partially aromatized small grains.

Figure 6 shows the intensity ratios for single grains with radii of 5 and $10 \AA$ for $U=1$ in some aromatic and aliphatic bands as a function of the aromatization degree, expressed through the parameter $E_{\text {gap }}$. The $I_{3.4} / I_{11.3}$ (red lines) and $I_{7} / I_{11.3}$ (green lines) ratios are most sensitive to $E_{\text {gap }}$, and are especially high for the $5 \AA$ grains. The $I_{3.4} / I_{3.3}$ ratio for the $5 \AA$ grains depends only weakly on $E_{\text {gap }}$, and this dependence essentially disappears for the $I_{3.4} / I_{3.3}$ ratio for the $10 \AA$ grains.

Further, we consider how various intensity ratios for an ensemble of grains corresponding to the J13 initial size distribution depend on external factors.

The dependences of the intensity ratios at different wavelengths after one million years on the UV intensity and the relative velocity of collisions between ions and dust grains $v_{\text {ion }}$ for $E_{0}=5 \mathrm{eV}$ are shown in Fig. 7 . The $I_{3.3} / I_{70+160}$ ratio can be used to trace variations in the radiation of small aromatic compounds relative to radiation of large grains. This ratio drops at $U<1$, then remains roughly constant. Figure 5a shows that the intensity in the $3.3 \mu \mathrm{m}$ band remains constant as $U$ increases, while the emission at 70 and $160 \mu \mathrm{m}$ varies. When the intensity of the radiation field increases, large dust grains are heated to higher temperatures, and the emission maximum is shifted from longer to shorter wavelengths. Accordingly, we first observe an increase in the intensity at 70 and $160 \mu \mathrm{m}$, after which this intensity remains constant. The ratio $I_{3.3} / I_{70+160}$ is also non-zero when $U=0.01$, but, in this case, we see emission of aliphatic rather than aromatic compounds, since the band at $3.4 \mu \mathrm{m}$ is nearby 
and fairly broad. A similar picture is observed for the $I_{7} / I_{70+160}$ ratio. Aliphatic compounds are responsible for the emission at $7 \mu \mathrm{m}$, but aromatic grains also have a band near this wavelength; therefore, generally speaking, it is not possible to distinguish one from the other for very low values of $U$, as in the previous case. However, as a result of the evolution, small aliphatic grains are absent for almost any $U$ value, so that the emission near $7 \mu \mathrm{m}$ usually reflects the composition of aromatic grains.

The $I_{3.4} / I_{11.3}$ ratio is the best tracer of the relative abundance of aliphatic and aromatic grains. This ratio is very high at low $U$ values, when all the grains are aliphatic, since the $11.3 \mu \mathrm{m}$ band is weak while the $3.4 \mu \mathrm{m}$ band is strong. This ratio then falls when aromatic grains appear in the medium, but not as much as it would in the absence of a contribution from the wings of the $3.3 \mu \mathrm{m}$ band. Thus, good quality spectra are essential for accurately separating the bands due to the different types of grains.

We also considered the dependence of the $I_{11.3} / I_{24}$ ratio on the UV radiation field. This ratio reaches a maximum at $U \approx 0.1$, and falls only slightly at lower values of $U$, since the aliphatic $11.3 \mu \mathrm{m}$ band is very weak. This decrease at small $U$ values is insignificant, since the $24 \mu \mathrm{m}$ emission also decreases with the intensity of the radiation field. The $I_{11.3} / I_{24}$ ratio drops stronger when $U$ is increased, due to an appreciable increase in the $24 \mu \mathrm{m}$ emission. A modest growth is again observed for high radiation fields $(U>100)$, due to the growth in the $11.3 \mu \mathrm{m}$-band intensity.

The blue curve in Fig. 7 shows the dependence of the mass fraction of small aromatic grains $q_{\mathrm{PAH}}$ on $U$. When $U<0.1, q_{\mathrm{PAH}}$ is modest, since the aromatization rate is low in the presence of such weak radiation, and not even the smallest grains are able to be constructed. When the radiation field is increased to $U=0.1$ or more after one million years, all small grains with sizes corresponding to PAHs become aromatic. In the presence of stronger radiation fields, this fraction may again decrease due to the destruction of small aromatic grains by UV radiation, but such strong fields can exist only in the immediate vicinities of hot stars.

Figure $7 \mathrm{~b}$ shows how these same ratios vary with the collisional velocity. It is obvious that they are much more sensitive to this parameter. When the collisional velocity between the ions and dust grains is $30 \mathrm{~km} / \mathrm{s}$, the fraction of small aromatic grains $\left(q_{\mathrm{PAH}}\right)$ becomes negligible after one million years. The 3.3 and $3.4 \mu \mathrm{m}$ emission bands are essentially absent by this time, causing the $I_{3.3} / I_{70+160}$ ratio to similarly drop sharply at velocities above 
$20 \mathrm{~km} / \mathrm{s}$. The $I_{7} / I_{70+160}$ and $I_{11.3} / I_{24}$ ratios decrease by an order of magnitude, but not as sharply as the $I_{3.3} / I_{70+160}$ ratio. This occurs because some contribution from large grains is possible both at 7 and $11.3 \mu \mathrm{m}$, while this is not the case at 3.3 and $3.4 \mu \mathrm{m}$.

In this study, we have considered only the emission properties of hydrocarbon grains. The shape of the continuum in the region of the considered aromatic and aliphatic bands can also be influenced by the presence of silicate absorption bands, which slightly change the considered ratios quantitatively. However, the qualitative behavior of these ratios remains the same. The detailed analysis of galaxy spectra from the SINGS survey presented in [44] did not detect significant indications of the presence of silicate absorption bands in nearly $90 \%$ of the studied galaxies. Our additional spectral computations show that these features begin to show up in emission only when $U=10^{4}-10^{6}$. This is consistent with the computational results of [9].

\section{CONCLUSION}

The presented results show that the evolution of an ensemble of dust particles strongly depends on the initial distributions of the dust size and aromatization degree. The J13 and MRN initial distributions that we have considered yield different results. The evolution of the J13 distribution, in which the mass fraction of small grains is initially large, is appreciably determined by the relative velocity of collisions with gas particles and the radiation field. In contrast, shattering processes play a key role in the MRN distribution, substantially reducing the fraction of large grains and increasing the mass fraction of small grains with time, partially compensating the influence of other factors that destroy small grains. The destruction of small grains by gas particles and radiation plays a "positive" role in preserving large grains in the J13 model, since one important factor leading to the destruction of large grains is shattering during collisions with smaller grains.

In the range of $U$ values considered, the radiation field chiefly leads to grain aromatization, while efficiently destroying only the smallest grains. Sputtering due to collisions with gas particles leads to the destruction of larger grains. Grains with sizes below $20 \AA$ are absent when the relative collision velocities are higher than $50 \mathrm{~km} / \mathrm{s}$. The destruction of grains will be even more efficient in the presence of higher velocities, such as are characteristic of supernovae. 
The IR emission spectrum of dust depends substantially on its evolution. The shape of the near-IR $(2-15 \mu \mathrm{m})$ spectrum of evolved dust in the case of high $E_{0}$ values is virtually independent of the intensity of the radiation field for $U$ values from 0.1 to $10^{4}$. Aromatization is not efficient at lower $U$ values, and characteristic aromatic bands are absent from spectra in this case. As the radiation field is increased, we observe a transition to spectra containing features characteristic for aromatic grains. The destruction of small grains is especially efficient for $\mathrm{C}-\mathrm{C}$ bond energies $E_{0}=2.9 \mathrm{eV}$. Even in the case of parameters of the medium characteristic of the "ordinary" ISM, small aromatic particles are completely absent in a medium with this $E_{0}$ value after one million years of evolution, leading to the absence of aromatic bands in its spectrum. Since these bands are preserved only when $E_{0}$ is about $5 \mathrm{eV}$, this indicates a preference for relatively high values of $E_{0}$.

The $I_{3.4} / I_{11.3}$ and $I_{7} / I_{11.3}$ intensity ratios demonstrate the greatest sensitivity to the aromatization degree of small grains. The $I_{3.4} / I_{3.3}$ ratio, which has often been proposed as a measure of the aromatization of small grains, is virtually independent of $E_{\text {gap }}$ for grains with radii of $5-10 \AA$. The $I_{3.4} / I_{11.3}$ ratio is a convenient indicator of the relative abundances of aliphatic and aromatic grains for various values of $U$ and $v_{\text {ion }}$. The $I_{3.3} / I_{70+160}$ ratio is a sensitive indicator of the contribution of aromatic grains to the total mass fraction of dust. The 3.3 and $3.4 \mu \mathrm{m}$ bands partially overlap, so that distinguishing each of them clearly requires good-quality observational data.

In this study, we considered only conditions that do not differ appreciably from those in the thermal phase of the ISM. It is obvious that the destruction of grains will be more efficient under the more extreme conditions inside regions of ionized hydrogen and in supernova remnants, which should be reflected in the corresponding dust emission spectra. Moreover, the spectra of the external radiation in such regions could differ from the spectrum considered in [21]. Shattering during high-velocity collisions between grains may not compensate for the destruction of small grains by the radiation field in this case, so that the corresponding spectra would differ from those presented here.

Our model can be used to consider various situations, including some not considered here. Its further application and optimization (especially the inclusion of grain charge, consideration of the evolution of silicate grains, and 3D computations) will be considered in future. The model can also be applied to studies of specific objects, such as IR bubbles around HII reagions, planetary nebulae, and supernova remnants. 


\section{ACKNOWLEDGEMENTS}

We thank the referee for important comments, and V. Akimkin and A. Jones for useful discussions. This work was supported by the Russian Foundation for Basic Research (grants 14-02-00604, 14-02-31456, 15-02-06204), a President of the Russian Federation Grant (MK4536.2015.2), and the "Dinastiya" foundation.

1. F. C. Gillett, W. J. Forrest, and K. M. Merrill, Astrophys. J. 183, 87 (1973).

2. A. Leger and J. L. Puget, Astron. Astrophys. 137, L5 (1984).

3. L. J. Allamandola, A. G. G. M. Tielens, and J. R. Barker, Astrophys. J. 290, L25 (1985).

4. S. Kwok and Y. Zhang, Astrophys. J. 771, 5 (2013).

5. W. W. Duley and A. Hu, Astrophys. J. 761, 115 (2012).

6. E. Churchwell, M. S. Povich, D. Allen, M. G. Taylor, M. R. Meade, B. L. Babler, R. Indebetouw, C. Watson, B. A. Whitney, M. G. Wolfire, T. M. Bania, R. A. Benjamin, D. P. Clemens, M. Cohen, C. J. Cyganowski, et al., Astrophys. J. 649, 759 (2006).

7. R. J. Simpson, M. S. Povich, S. Kendrew, C. J. Lintott, E. Bressert, K. Arvidsson, C. Cyganowski, S. Maddison, K. Schawinski, R. Sherman, A. M. Smith, and G. Wolf-Chase, Mon. Not. R. Astron. Soc. 424, 2442 (2012).

8. Y. N. Pavlyuchenkov, M. S. Kirsanova, and D. S. Wiebe, Astron. Rep. 57, 573 (2013).

9. B. T. Draine and A. Li, Astrophys. J. 657, 810 (2007).

10. K. M. Sandstrom, A. D. Bolatto, B. T. Draine, C. Bot, and S. Stanimirović, Astrophys. J. 715, 701 (2010).

11. M. S. Khramtsova, D. S. Wiebe, P. A. Boley, and Y. N. Pavlyuchenkov, Mon. Not. R. Astron. Soc. 431, 2006 (2013).

12. C. W. Engelbracht, K. D. Gordon, G. H. Rieke, M. W. Werner, D. A. Dale, and W. B. Latter, Astrophys. J. 628, L29 (2005).

13. D. S. Wiebe, M. S. Khramtsova, O. V. Egorov, and T. A. Lozinskaya, Astron. Lett. 40, 278 (2014).

14. M. S. Khramtsova, D. S. Wiebe, T. A. Lozinskaya, and O. V. Egorov, Mon.Not. R. Astron. Soc. 444, 757 (2014). 
15. A. P. Jones, L. Fanciullo, M. Köhler, L. Verstraete, V. Guillet, M. Bocchio, and N. Ysard, Astron. Astrophys. 558, A62 (2013).

16. M. S. Murga, S. A. Khoperskov, and D. Z. Wiebe, Astron. Rep. 60, 233 (2016).

17. H. Hirashita, Mon. Not. R. Astron. Soc. 407, L49 (2010).

18. H.-P. Gail and E. Sedlmayr, Physics and Chemistry of Circumstellar Dust Shells (Cambridge Univ. Press, Cambridge, UK, 2014).

19. J. E. Chiar, A. G. G. M. Tielens, A. J. Adamson, and A. Ricca, Astrophys. J. 770, 78 (2013).

20. D. A. García-Hernández and S. K. Górny, Astron. Astrophys. 567, A12 (2014).

21. J. S. Mathis, P. G. Mezger, and N. Panagia, Astron. Astrophys. 128, 212 (1983).

22. W. W. Duley, Astrophys. Space Sci. 23, 43 (1973).

23. K. Sellgren, M. W. Werner, and H. L. Dinerstein, Astrophys. J. 271, L13 (1983).

24. B. T. Draine and A. Li, Astrophys. J. 551, 807 (2001).

25. Y. N. Pavlyuchenkov, D. S. Wiebe, V. V. Akimkin, M. S. Khramtsova, and T. Henning, Mon. Not. R. Astron. Soc. 421, 2430 (2012).

26. A. P. Jones, Astron. Astrophys. 540, A1 (2012).

27. A. P. Jones, Astron. Astrophys. 540, A2 (2012).

28. A. P. Jones, Astron. Astrophys. 542, A98 (2012).

29. A. P. Jones, Astron. Astrophys. 545, C2 (2012).

30. A. P. Jones, Astron. Astrophys. 545, C3 (2012).

31. N. V. Voshchinnikov, V. B. Il'in, and T. Henning, Astron. Astrophys. 429, 371 (2005).

32. B. T. Draine and H. M. Lee, Astrophys. J. 285, 89 (1984).

33. B. T. Draine and H. M. Lee, Astrophys. J. 318, 485 (1987).

34. P. Pilleri, C. Joblin, F. Boulanger, and T. Onaka, arXiv:1502.04941 [astro-ph.GA] (2015).

35. T. I. Mori, T. Onaka, I. Sakon, D. Ishihara, T. Shimonishi, R. Ohsawa, and A. C. Bell, Astrophys. J. 784, 53 (2014).

36. C. Joblin, A. G. G. M. Tielens, L. J. Allamandola, and T. R. Geballe, Astrophys. J. 458, 610 (1996).

37. H. Hirashita and H. Yan, Mon. Not. R. Astron. Soc. 394, 1061 (2009).

38. H. Yan, A. Lazarian, and B. T. Draine, Astrophys. J. 616, 895 (2004).

39. J. S. Mathis, W. Rumpl, and K. H. Nordsieck, Astrophys. J. 217, 425 (1977).

40. D. Massa and B. Savage, in Interstellar Dust, Ed. by L. J. Allamandola and A. G. G. M. Tielens, 
IAU Symp. 135, 3 (1989).

41. R. Siebenmorgen, N. V. Voshchinnikov, and S. Bagnulo, Astron. Astrophys. 561, A82 (2014).

42. C. W. Engelbracht, K. D. Gordon, G. H. Rieke, M. W. Werner, D. A. Dale, and W. B. Latter, Astrophys. J. 628, L29 (2005).

43. B. T. Draine, D. A. Dale, G. Bendo, K. D. Gordon, J. D. T. Smith, L. Armus, C. W. Engelbracht, G. Helou, R. C. Kennicutt, Jr., A. Li, H. Roussel, F. Walter, D. Calzetti, J. Moustakas, E. J. Murphy, et al., Astrophys. J. 663, 866 (2007).

44. J. D. T. Smith, B. T. Draine, D. A. Dale, J. Moustakas, R. C. Kennicutt, Jr., G. Helou, L. Armus, H. Roussel, K. Sheth, G. J. Bendo, B. A. Buckalew, D. Calzetti, C. W. Engelbracht, K. D. Gordon, D. J. Hollenbach, et al., Astrophys. J. 656, 770 (2007). 